\title{
Design and performance of a pulsed power-driven $x$-ray source for flash radiography
}

\author{
Xie Weiping, Xia Minghe, Guo Fan, Geng Lidong, Zhao Yue, Xu Le, Feng Shuping, \\ Zhou Liangji, Wei Bing, He An, Yuan Jianqiang, Chen Lin, Li Hongtao, Han Wenhui, \\ Jiang Jihao, Li Feng, Wang Zhi, Li Yong, Kang Junjun, Zhang Yuanjun, Wu Wei, \\ Wang Meng, and Zou Wenkang \\ Institute of Fluid Physics, China Academy of Engineering Physics, Mianyang 621000, China
}

(Received 31 March 2021; accepted 13 October 2021; published 15 November 2021)

\begin{abstract}
The design and test results of a pulsed power-driven X-ray source which is named Hawkeye-I are described. The Hawkeye-I is developed to execute flash X-ray radiography. It is a six-stage induction voltage adder and terminated by a positive polarity rod-pinch diode. The six stages are identical and the prime energy of each stage is provided by a tesla transformer elevating the output voltage from a discharge brick which consists of two $4 \mu \mathrm{F}$ capacitors and a three-electrode gas switch. Comparing to the induction voltage adders driven by Marx generators, the numbers of gas switches and capacitors in the energy storage sections of Hawkeye-I decrease significantly. The Hawkeye-I can produce a maximum output voltage of about 4.2 MV. The corresponding X-ray source spot size and dose at $1 \mathrm{~m}$ are $1.4 \mathrm{~mm}$ and $17.9 \mathrm{rad}$, respectively. Fifty consecutive shots have been conducted to evaluate the reliability of the machine and $98 \%$ of the shots are successful. Furthermore, as the energy propagating in the accelerator is mainly determined by seven lasertriggered gas switches, the Hawkeye-I is capable of controlling the timing of the X-ray pulse accurately.
\end{abstract}

DOI: $10.1103 /$ PhysRevAccelBeams.24.110401

\section{INTRODUCTION}

Flash X-ray radiography has been used extensively in hydrodynamic experiments to take stop-action images of the materials driven by explosives. It is an essential diagnostic to obtain internal interface locations and density distribution information which enable to improve the models of the material's responses to impulsive loading such as crack formation and propagation, spall, and ejecta [1-3]. Electron beam diode driven by pulsed power machine is a way to obtain the flash $\mathrm{X}$ ray that takes advantages of low cost and compactness. At present, many pulsed power accelerators are being built and executed around the world for flash radiography programs, such as radiographic integrated test stand (RITS) [4] and Cygnus [5-7] in the United States, SuperSwarf [8] and Merlin [9] in the United Kingdom, Asterix [10] in France, and Jianguang accelerators [11,12] in China.

In recent years, due to the requirement of executing the hydrodynamic experiments in our laboratory, we have

\footnotetext{
*Corresponding author. 394560789@qq.com

Published by the American Physical Society under the terms of the Creative Commons Attribution 4.0 International license. Further distribution of this work must maintain attribution to the author(s) and the published article's title, journal citation, and DOI.
}

completed a pulsed power-driven X-ray source which is named Hawkeye-I to provide the high dose and small spot size $\mathrm{X}$ rays. The expected dose at $1 \mathrm{~m}$ from the load should be greater than $13 \mathrm{rad}$ and the spot size less than $1.6 \mathrm{~mm}$ is in demand. As hydrodynamic experiments are usually high-value events, the high reliability of this accelerator is necessary. The reliability is specified at better than one failure out of 50 shots. Moreover, the firing of the machine must be able to synchronize with the actions of explosives to obtain images at a specific moment [13-17].

The Hawkeye-I is composed of six identical stages and terminated by a rod-pinch diode (RPD). The pulsed power source of each stage mainly consists of an energy storage section, a tesla transformer, an intermediate storage capacitor (ISC), a laser triggered gas switch (LTGS), a pulse forming line (PFL), a self-breaking oil switch, and one water transmission line (WTL). The voltage pulses produced by each source are added by six induction cavities and the summed voltages transmit to the RPD through an output transmission line.

In this paper, we will present the design details of the main components of Hawkeye-I, such as tesla transformer, ISC, output transmission line, and RPD. The contents will be shown in the following section. In Sec. III, we will give the experimental results. The operating reliability and synchronization ability of this machine are evaluated which will be also described in this section. Section IV presents conclusions. 


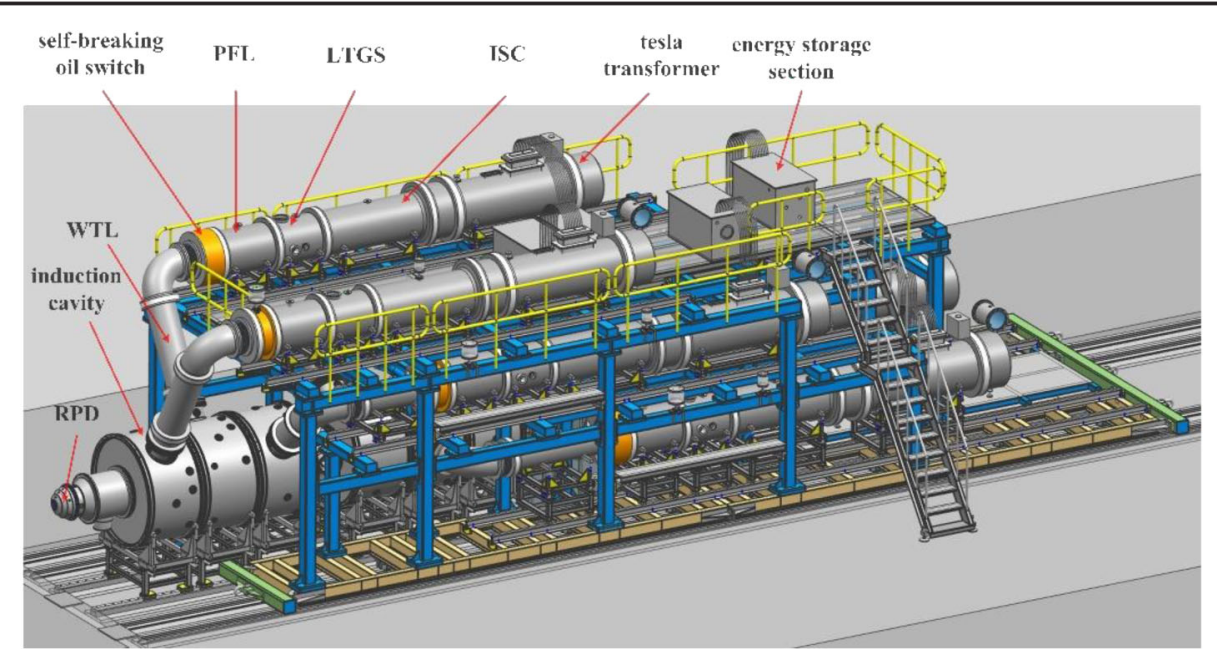

FIG. 1. Overview of the Hawkeye-I. The six stages are divided into three layers and each layer includes two pulsed power sources. The six induction cells are deployed at the central region and terminated by an RPD.

\section{DESIGN OF THE MACHINE}

Figure 1 shows the schematic view of the Hawkeye-I. An energy storage section that consists of two $4 \mu \mathrm{F}$ capacitors and a three-electrode gas switch is employed in each stage to supply the prime energy. The output pulse from this section is elevated by a tesla transformer and then charges the ISC whose capacitance and one-way transmit time are $13.4 \mathrm{nF}$ and $53.6 \mathrm{~ns}$, respectively. The ISC is switched out by an LTGS which is a key component to synchronize the pulses feeding the induction cells. It also reduces the rise time of the voltage pulse across PFL. The impedance and one-way transmit time of each PFL are $4 \Omega$ and $27 \mathrm{~ns}$, respectively. The self-breaking oil switch is used to discharge the PFL to a WTL with $7 \Omega$ impedance and $70 \mathrm{~ns}$ one-way transmit time. As the parasitic capacitance of the self-breaking oil switch is much less than that of the water switch, a prepulse switch is not incorporated in Hawkeye-I. The voltage pulses transmitting through the self-breaking oil switches and propagating along the WTLs are added by the induction cavities, and the summed voltages are restricted to the output transmission line region. The output transmission line is in positive polarity since the positive polarity RPD could produce the X-ray source with a smaller spot size than that of negative polarity one [18-20]. It also should be mentioned that the output transmission line operates as a lossless vacuum insulation line, i.e., the electric stress on its cathode surface is lower than the explosive electron emission threshold. This is also required to obtain the preferred X-ray source because the electron flow in the magnetically insulated transmission line is very harmful to the RPD performance [21-23].

\section{A. Pulsed power source}

The prime energy of each pulsed power source is provided by an energy storage section and a tesla transformer which are shown in Fig. 2. The two capacitors and a three-electrode gas switch in the energy storage section are arranged with the same configuration as a brick in the linear transformer driver [24]. Once the conduction of the gas switch is induced by an external voltage pulse, the energy stored in capacitors is transmitted to the tesla transformer through ten identical feed-in cables. The impedance and length of each feed-in cable are $75 \Omega$ and $1.5 \mathrm{~m}$, respectively. As shown in Fig. 2, the tesla transformer has a cylindrical configuration whose length and outer diameter are 3 and $0.96 \mathrm{~m}$, respectively. Its main components include an outer shell, an inner conductor, two magnetic cores, two plastic diaphragms, a primary coil, and a secondary coil. The magnetic cores are fabricated with silicon steel and the plastic diaphragms are made of high-density polyethylene (HDPE). The primary coil consists of two turns which are wound with copper wires on an HDPE framework of cylindrical configuration. One end of the primary coil is connected with the inner conductors of the ten feed-in

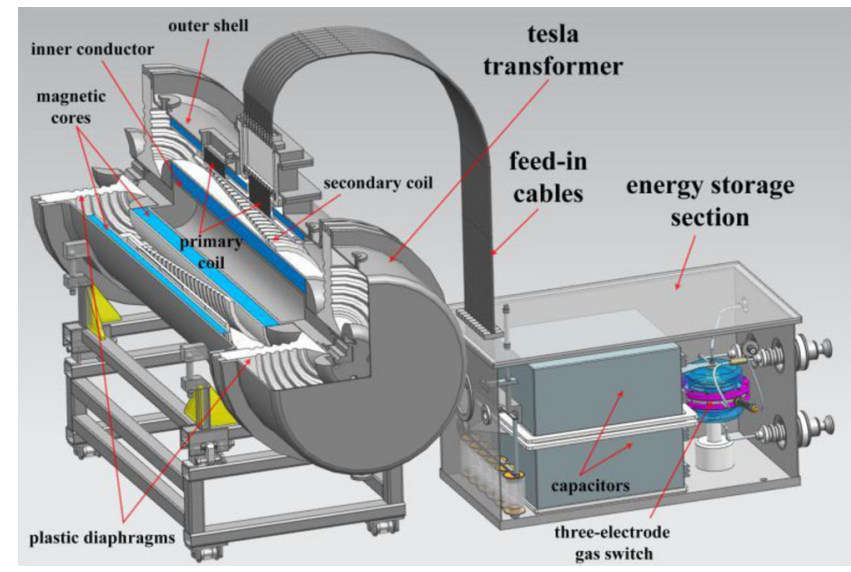

FIG. 2. Schematic view of the energy storage section and tesla transformer. Both the volumes of the energy storage bank and tesla transformer are filled with transformer oil. 
cables and the other end together with the outer conductors of the feed-in cables are joined with the tesla transformer outer shell. The secondary coil includes 21.5 turns which are wound with the same copper wires along an HDPE framework of hollow cone configuration. The two ends of the secondary coil are connected to the outer and inner conductors of the tesla transformer, respectively. More detailed descriptions of the energy storage section and tesla transformer are presented in Ref. [25].

The conductions of the six three-electrode gas switches in the energy storage sections are controlled by the electrical pulses from a trigger unit which is displayed in Fig. 3. The trigger unit is made up of two $20 \mathrm{nF}$ capacitors, a gas switch, and six $75 \Omega / 250 \mathrm{kV}$ transmission cables. The capacitors and gas switch are also located in a transformer oil tank and the discharging circuit topology is the same as that discussed above. The gas switch is triggered by an Nd:YAG laser which is capable of producing $\sim 60 \mathrm{~mJ}$ output energy at the $266 \mathrm{~nm}$ wavelength. The laser beam enters the switch via a polymethyl methacrylate (PMMA) tube. Six transmission cables transmit the outputs of the trigger unit to the six three-electrode gas switches. Figure 4 displays the typical output voltage of the trigger unit on the condition that the six cables are all terminated by the matched resistor loads.

Because the output pulse of the tesla transformer has a very long rise time, a pulse forming system as shown in Fig. 5 is employed to compress its rise time and pulse width. The ISC is a water dielectric coaxial line and the

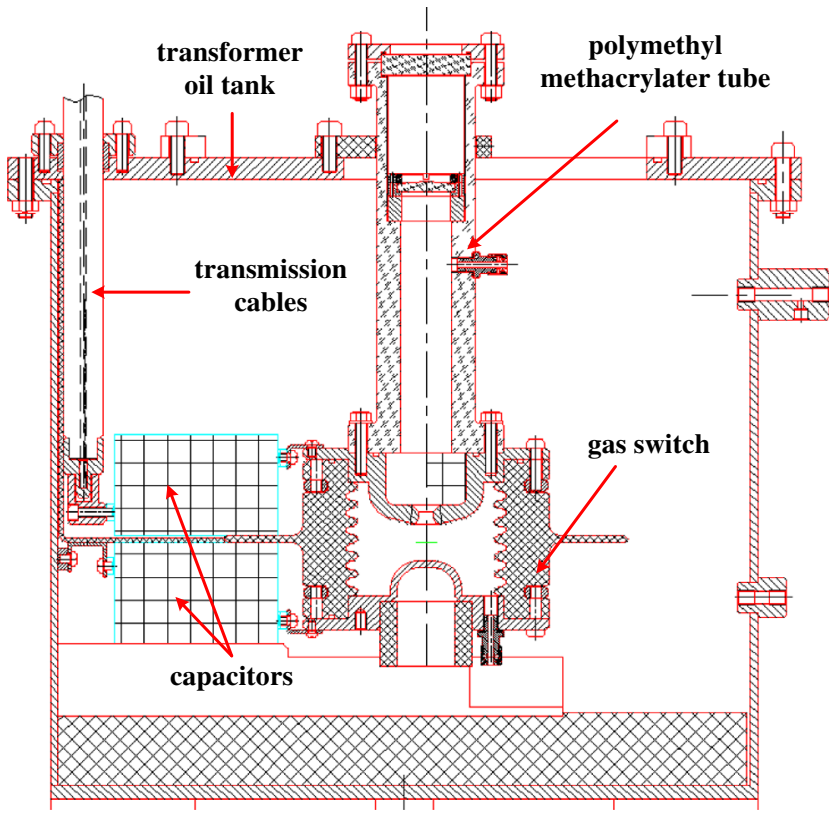

FIG. 3. The layout of the trigger unit. A resistor divider is fielded between one end of the upper capacitor and the transformer oil tank shell to diagnose the output of the trigger unit. The gas switch uses dry air with a gas pressure of about $0.38 \mathrm{MPa}$. Its working ratio is roughly $43 \%$ and the jitter is only about 2 ns.

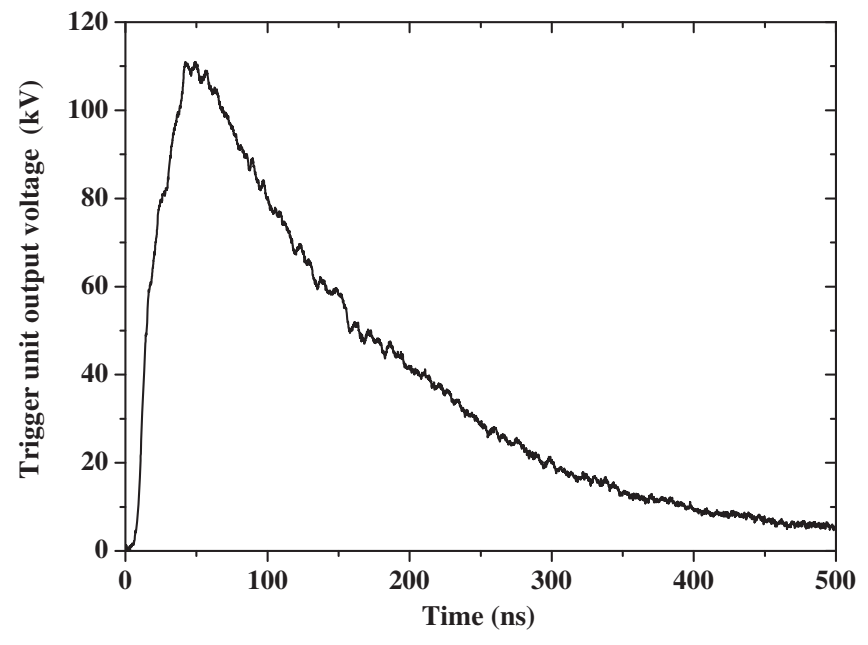

FIG. 4. The output voltage of the trigger unit. The maximum value and $10-90 \%$ rise time are about $110 \mathrm{kV}$ and $27.3 \mathrm{~ns}$, respectively.

radii of its outer and inner conductors are 35 and $19.2 \mathrm{~cm}$, respectively. For the nominal shot, the $0-100 \%$ rise time of the voltage pulse on ISC is about $3.8 \mu \mathrm{s}$ which is much longer than those of pulsed power machines driven by Marx generators. The effective pulse duration, defined as the time during which the voltage is above $63 \%$ of the peak value, is $1.73 \mu \mathrm{s}$, and also increases a lot. It decides the maximum allowed electrical stress in the ISC. Therefore, the electrical strength in this component must be designed at a lower level than those of other accelerators. According to the empirical formula proposed by Martin, the breakdown field in the bulk of water could be easily calculated [26]. The actual stress on the ISC inner conductor which is in negative polarity is about $52 \%$ of the breakdown field. Meanwhile, the breakdown fraction for the outer conductor is $60.4 \%$.

The flashover occurring over the interfaces between the plastic diaphragms and water in ISC is a more serious concern that should be taken into account carefully [27]. Whereas, the safety electrical stress can neither be quoted directly from the data gained before nor be obtained by a well-known theory. During the initial development of Hawkeye-I, we have designed a prototype of ISC. Figure 6 depicts the electrical stresses on the ISC plastic barriers surfaces. The barriers are made of HDPE and the ISC is successfully used more than 1000 shots at $110 \%$ of the nominal operating voltage. However, after assembling all components and starting commissioning of the Hawkeye-I, electrical breakdown along the diaphragmwater interface takes place occasionally. This may be attributed to the material performance variation.

It is seen from Fig. 5 that the diameter of the ISC at the upstream end is slightly larger than that at the downstream side. The maximum stress on the upstream plastic barrier is also somewhat low. The surface tracks always happen on 


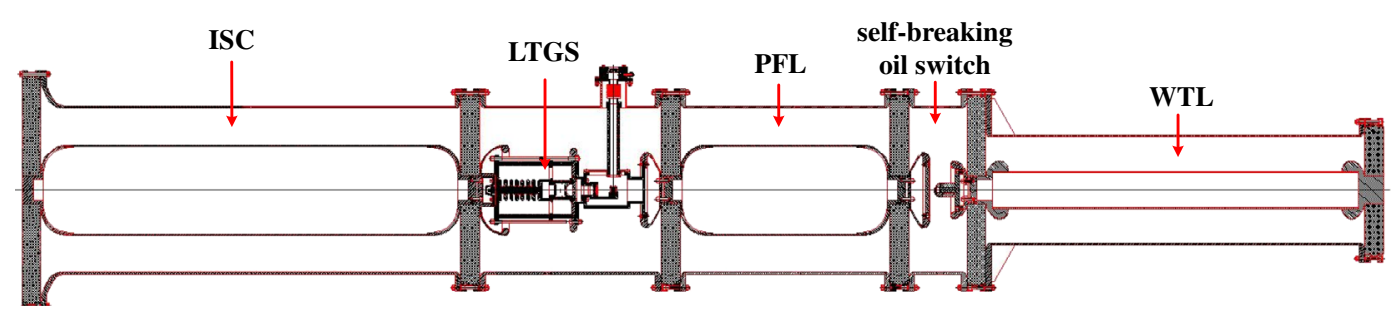

FIG. 5. Cross-sectional view of the pulse forming system. The ISC, PFL, and WTL are filled with deionized water. The regions accommodating the LTGS and self-breaking oil switch are full of transformer oil.

the downstream one. According to the experimental results given by Ushakov, the insulators made of nylon exhibited slightly higher flashover voltage than those made of HDPE [28]. The plastic barriers of all six ISCs in Hawkeye-I are substituted by those made of nylon. The six ISCs have succeeded more than 100 shots. We have examined the plastic barriers with an endoscope over each group of ten consecutive shots. No evidence of surface track was observed.

The LTGS serves as the last command-fired switch of each PFL and mainly determines the driven sequence of the induction cells. It is $39.5 \mathrm{~cm}$ in length, $28.4 \mathrm{~cm}$ in diameter, and is filled with $\mathrm{SF}_{6} / \mathrm{N}_{2}$ mixture gas at a gas pressure of about $0.15 \mathrm{MPa}$. The LTGS has a main triggered portion followed by seven identical self-breakdown sections. The triggered section consists of two hemispherical electrodes which are separated by a cylindrical PMMA chamber of $3.2 \mathrm{~cm}$ in length. The adjacent electrodes in the selfbreakdown portions are separated by the PMMA rods each with a length of $1.3 \mathrm{~cm}$. All of those components are enveloped in a PMMA chamber of $22 \mathrm{~cm}$ in diameter.

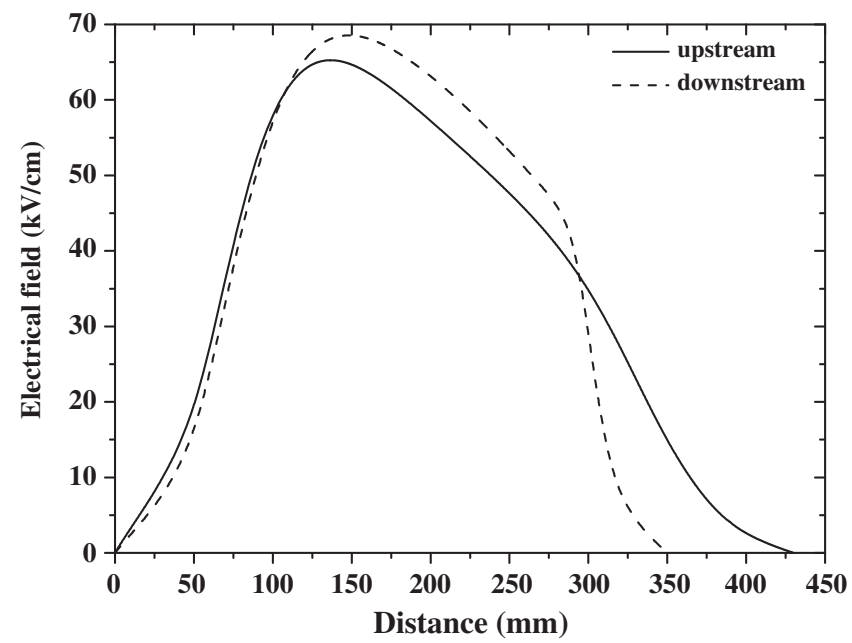

FIG. 6. Electrical fields distribution on the diaphragms-water interfaces of the ISC. The solid line indicates the stresses at the upstream end, while the dashed line denotes the fields at the downstream side. The peak fields on the upstream and downstream plastic barriers are 65.2 and $68.7 \mathrm{kV} / \mathrm{cm}$, respectively. Those values are lower than the maximum stress on the ISC barriers of the RITS accelerator [4].
Each LTGS is triggered by the same kind of laser facility for controlling the trigger unit. The laser beam from the laser facility is in parallel with the axis of the LTGS. It is deflected at an angle near $90^{\circ}$ by a quartz glass and then enters into a metal housing where a prism is located. The laser beam will be deflected again by this prism. An aperture with a diameter of $0.8 \mathrm{~cm}$ in one of the electrodes of the triggered portion lets the laser beam transmit into the triggered gap. Finally, the laser beam is focused near the middle of the gap by a focusing optic. Both the prism and focusing optic are made of calcium fluoride, as they have the feature of resistance to the debris from discharging product of $\mathrm{SF}_{6}$ and exhibit a lifetime of over 100 shots [29].

The synchronization of the Hawkeye-I with the ignition of explosives is mainly determined by the actions of the six LTGSs and the gas switch in the trigger unit. Those switches are triggered by seven Nd:YAG lasers that are controlled remotely by another system as displayed in Fig. 7. A central control unit that supplies the triggering commands for all activities gives an optical signal and

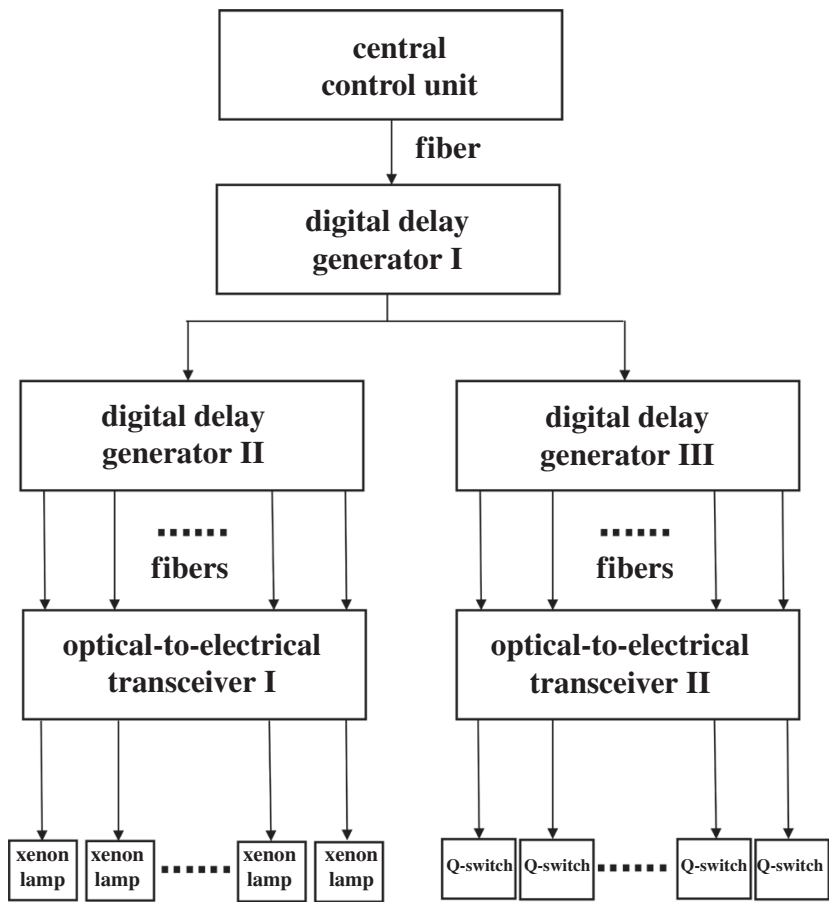

FIG. 7. Block diagram of the control system of the seven lasers. 
triggers the first digital delay generator (DDG). This DDG produces two signals and controls the other two DDGs. The optical signals from the second DDG are transmitted into an optical-to-electrical transceiver via fiber where the optical signals are converted into low voltage electrical outputs. The xenon lamps of the seven lasers will be triggered by those electrical signals. The Q-switches of the seven lasers are controlled by the third DDG in the same way.

The self-breaking oil switch has a rod-plane configuration. The distance between the rod and plane electrode could be adjusted to accommodate different voltage pulses. For the nominal operation, the maximum voltage across the self-breaking oil switch is about 1.4 MV and the distance is set to be $17 \mathrm{~mm}$. Since the effective pulse durations of the voltage pulses on PFL and WTL are approximately the same as those of existing induction voltage adders (IVAs), their detailed design methods and configurations are therefore not elaborated in this article.

\section{B. Voltage adder section}

The voltage pulses generated by the six pulsed power sources are summed at the voltage adder section which is displayed in Fig. 8. The induction cavities are electrically connected together whose central areas are threaded by a continual inner conductor forming the output transmission line. The inner stalk is cantilevered from a basement at the left end of the first cavity which is not shown in Fig. 8. The stalk is made of stainless steel and divided into two segments. According to the mechanical analysis, a maximum deformation of $4.79 \mathrm{~mm}$ will be produced at the far downstream end due to its own gravity. We have employed two wedge shims between the two segments to compensate for the deflection [30]. By careful adjustment, the linearity of the inner stalk is within $\pm 0.5 \mathrm{~mm}$, and the misalignment between the outer conductors and inner electrode of the output transmission line is less than $\pm 2 \mathrm{~mm}$.
The induction cavity is mainly composed of a feed port, an azimuthal transmission line, an insulation stack, magnetic cores, and a cathode plate. The voltage generated by the pulsed power source transmits into the induction cavity through the feed port and then flows in two opposite directions along the azimuthal transmission line. A halfcircle continuous tab opposite the feed port allows current conduction from the azimuthal transmission line to the cathode plate. The cathode plate is employed to reduce the cell inductance and help grade the voltage uniformly on the insulator rings. The maximum electric stress on the cathode plate surface is designed to be $200 \mathrm{kV} / \mathrm{cm}$. It is made of aluminum alloys and the hard-anodized process is applied to form dielectric films on its surface that is an effective way to enhance the explosive electron emission threshold [31]. Although the threshold higher than $300 \mathrm{kV} / \mathrm{cm}$ could be acquired, part of dielectric films on the cathode plate surface are found to be abraded over hundreds of shots despite all of the individual abraded areas are within $1 \mathrm{~cm}$ in diameter. This might be due to the bombardment of debris generated from the diode since the broken areas are always at the bottom of the cathode plate. In addition, the condition of the insulation stack at the bottom region is also worse than other places and many flashover marks are founded underneath over tens of shots. More detailed descriptions of other components in the induction cell are given in Ref. [32].

For an ideal IVA, the output transmission line is designed with a stepped impedance profile along the power flow direction either in driver-matched or load-matched mode, and the cells are fired synchronously with the speed at which the electromagnetic wave propagates along the line $[33,34]$. However, because of the requirement of a lossless vacuum insulation state, the impedances of the output transmission line increase linearly from $20 \Omega$ at the first cell to $70 \Omega$ at the last one. The maximum electrical field on the output transmission line cathodes surfaces is $150 \mathrm{kV} / \mathrm{cm}$.

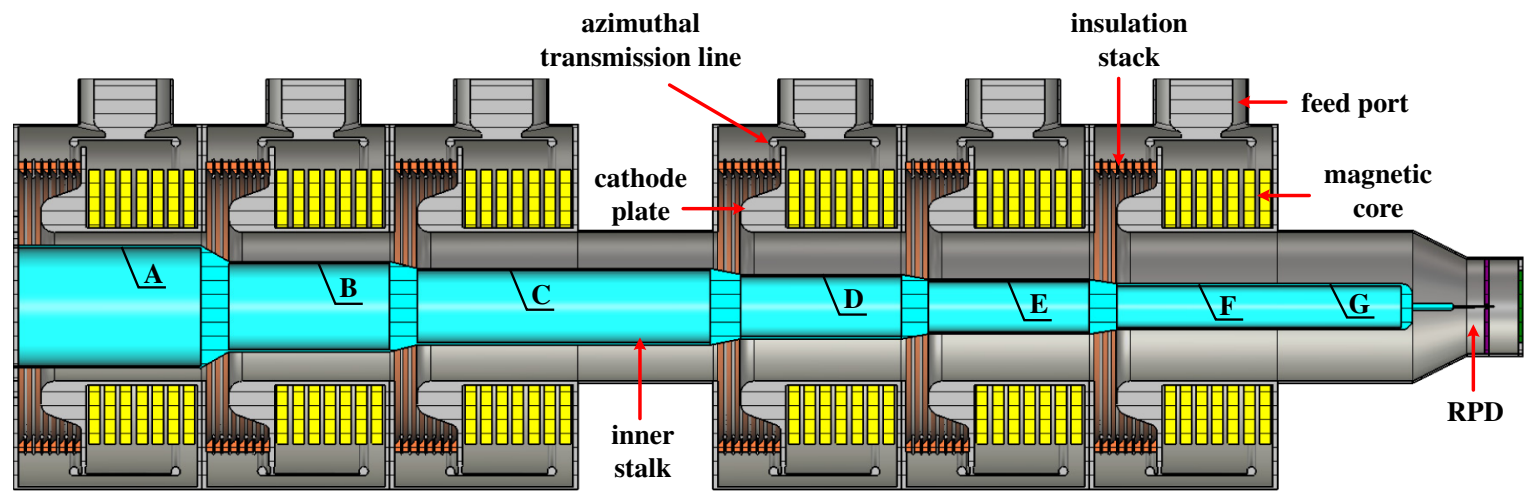

FIG. 8. Schematic cross-sectional view of the voltage adder system which consists of six single feed port induction cavities and an inner stalk. The six cells are divided into two groups. Two coaxial tubes are employed between the third and fourth cavities and at the end of the last cell to accommodate vacuum pumps. A-F indicate the positions of D-dot sensors for detection of the output transmission line voltages. $\mathrm{G}$ represents the location of D-dot and B-dot monitors for voltage and current measurement downstream of the last induction cell which is about $45 \mathrm{~cm}$ from the RPD. All of these sensors are installed on the inner stalk. 
The cathodes are also made of hard-anodized aluminum alloys. Their inner diameters are $66 \mathrm{~cm}$ and large enough to allow a person to get in for in-suit inspection and cleaning the insulation stack.

Another concern that should be taken into account is the anomalous voltages resulting from the nonideal driven sequence of the induction cells $[35,36]$. Because of the jitters of LTGS and self-breaking oil switch, the induction cavity is unlikely to be driven exactly at the time when the electromagnetic wave arrives at this cell. The induction cavity driven earlier than the desired timing will induce a higher voltage than the nominal value, while the voltage across the cell fired later becomes low and even a reverse polarity voltage appears. The induction cells must be able to withstand the anomalous voltages of the worst-case driven sequence. We have proposed a full circuit model of Hawkeye-I to investigate this issue. The circuit model indicating one of the six stages of the machine is validated according to the experimental results [32,37]. Tests of a pulse forming section demonstrate that the jitters of LTGS and self-breaking oil switch are about 3 and 4 ns, respectively. A random number generator is used to produce the firing times of the six cavities which are incorporated into the circuit simulation model. The simulation results suggest that the maximum voltage across each induction cavity is nearly identical and about $1.0 \mathrm{MV}$ if the six cells are driven with ideal timing, and the nonideal driven sequence will increase the voltage to $1.3 \mathrm{MV}$ to get the same peak load voltage.

\section{Rod-pinch diode}

Since the researchers from Naval Research Laboratory invented the RPD, it has been successfully fielded on many pulsed power accelerators and proved to be a promising Bremsstrahlung radiation source for flash radiography [38]. The RPD is employed at the end of the voltage adder section which is also depicted in Fig. 8. It is made up of a tungsten anode rod and an aluminum cathode plate with an annular aperture at its central region. The anode rod with a blunt tip extends $14 \mathrm{~mm}$ beyond the annular cathode aperture. The diameters of the anode rod and cathode aperture are 1.5 and $18 \mathrm{~mm}$, respectively.

The electron beam pinch formation in RPD is similar to other diodes studied previously. When the electric field on the cathode exceeds the explosive electron emission threshold, the plasma will be produced on the cathode surface and electrons emission from the cathode will be initialized. During this stage, electrons propagate radially from the cathode to anode and the RPD operates in the space charge limited flow. As the diode voltage and current increase, the self-induced magnetic field of the current bends the electron trajectories. The electrons begin to flow toward the anode tip. After sufficient energy is deposited on the anode rod, surface contaminants are desorbed and subsequently ionized, forming a plasma over the anode surface. The ions will be extracted from anode plasma and help the electrons efficiently pinch to the anode rod tip, forming the magnetically limited flow [18-20,39]. The anode and cathode plasmas expand toward each other during the discharge period. This reduces the effective ratio of the cathode to anode radii and consequently resulting in diode impedance decaying. The impedance collapses when the diode anodecathode (A-K) gap is shortened by the plasmas.

The X-ray yield from the RPD is strongly influenced by the plasmas behaviors, and the misalignment between the anode rod and cathode plate of the RPD affects the spending time of the plasmas shorting the A-K gap. An electrode concentricity evaluation system (ECES) is employed during the refurbishment of the diode to make the anode rod and cathode plate as homocentric as possible [40]. The ECES sets a camera in the front of the diode to capture the images of the anode rod and cathode plate which are displayed on a computer in real time. The cathode plate is adjusted to align with the anode rod by this guide. The concentricity between the anode rod and cathode aperture within $0.05 \mathrm{~mm}$ could be obtained. A PMMA plate is used to seal the vacuum chamber which allows the ECES to monitor the status of the anode rod and cathode aperture when pumping the vacuum chamber. It is observed that the concentricity usually deteriorates after vacuum pumping and the misalignment increases to $0.3 \mathrm{~mm}$. This may result from the deformation of the O-rings introduced between adjacent induction cavities. Nevertheless, the RPD operates as expected without degradation of the X-ray dose evidently at this extent.

\section{EXPERIMENTAL RESULTS}

\section{A. Performance of the machine}

Figure 9 depicts the typical voltage waveform of the ISC component which is measured by a D-dot sensor at the middle of this component. For this shot, the capacitors in

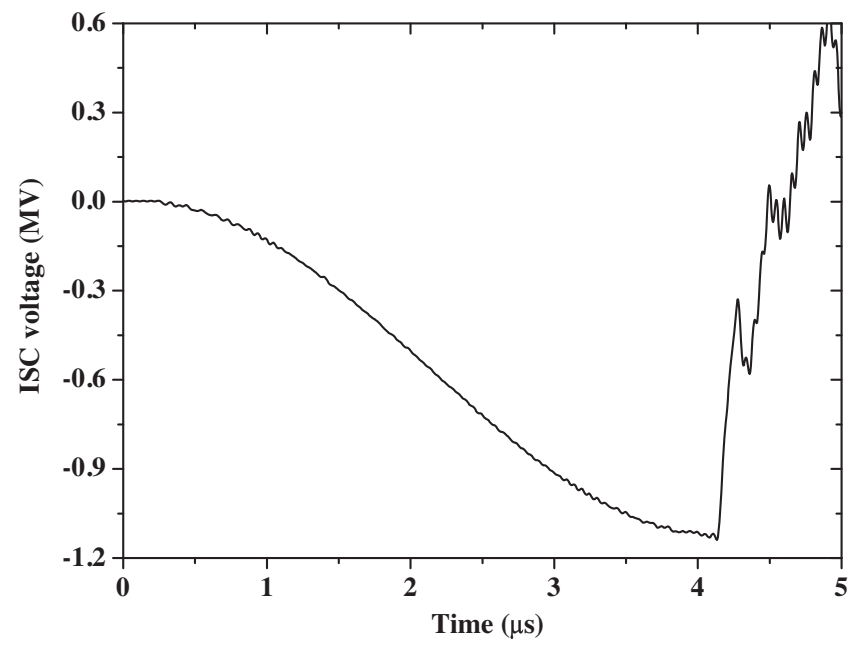

FIG. 9. The voltage pulse on ISC component. 


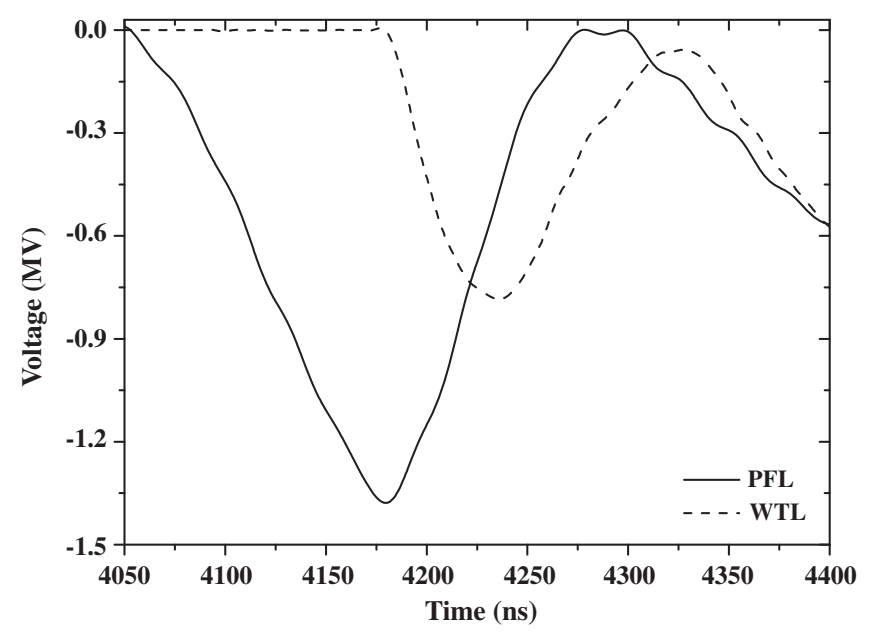

FIG. 10. Voltage waveforms of the PFL (solid line) and WTL (dashed line). The PFL voltage was obtained by a D-dot probe at the middle of this component while the voltage pulse on WTL was monitored by another D-dot sensor at the front of this element.

the energy storage sections were charged at $\pm 60 \mathrm{kV}$. The amplitude of the voltage pulse was $1.14 \mathrm{MV}$. Thus, the actual transformation ratio of the tesla transformer should be 9.5. The voltage pulse on PFL is exhibited in Fig. 10 whose peak value is $1.38 \mathrm{MV}$. The charging time of the PFL was $130 \mathrm{~ns}$, and the self-breaking oil switch closed nearly 20 ns before the PFL was charged to the maximum value. Although the equivalent capacitance of the ISC is much greater than the PFL capacitance, the PFL voltage is only slightly higher than that of the ISC. The energy might be dissipated in the LTGS. In fact, circuit simulation is employed to reproduce the experimental results. It suggests that the resistance of the LTGS during the conduction state is about $0.5 \Omega$. The forward traveling voltage wave generated by the PFL is also displayed in Fig. 10. The maximum value and $10-90 \%$ rise time were about $785 \mathrm{kV}$ and $32.5 \mathrm{~ns}$, respectively.

We have measured the operating voltages of each induction cavity. The typical results are shown in Fig. 11. Those waveforms indicate the driven sequence of the six cells. For this shot, the No. 1 cavity which was near the ground end of the voltage adder section was driven firstly, and the No. 6 cell close to the RPD was fired lastly. It is seen that the peak voltages of the induction cells deviate from each other. The maximum values were about 1.1 MV for the first group of three cavities, while the amplitude of the voltage pulse on the No. 6 cell was the lowest and only about $850 \mathrm{kV}$.

Figure 12 presents the summed voltages within the output transmission line region. The amplitudes at each induction cell section were 1.04, 2.11, 3.12, 3.57, 3.84, and 4.36 MV, respectively. It demonstrates that the voltages from the pulsed power sources are added by the induction cavities as expected. However, it is observed that the first group of three induction cells exhibit a much higher voltage

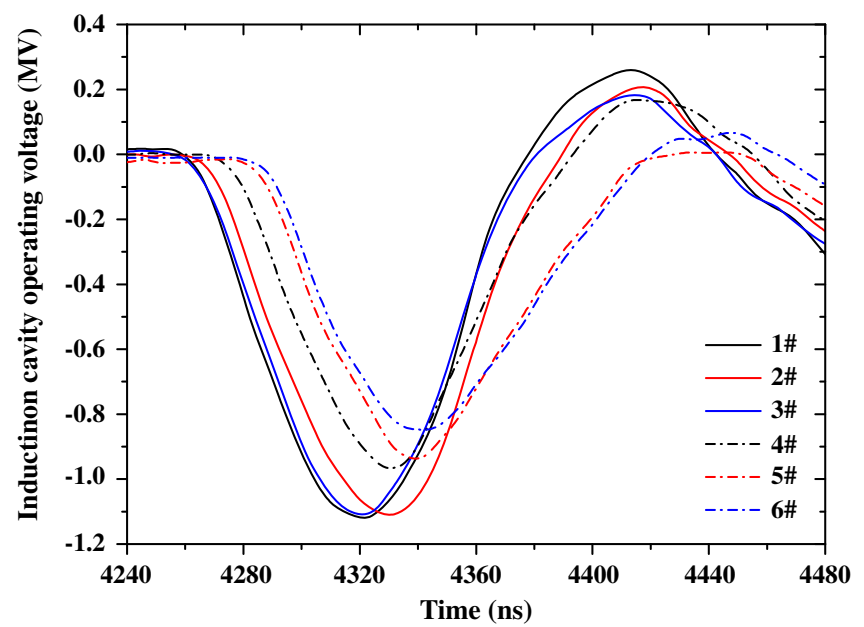

FIG. 11. Voltage waveforms of the six induction cavities. They were acquired by the D-dot probes at the center of the feed port sections.

adding efficiency than that of the second group. This is mainly due to that not only the operating voltages of the induction cavities themselves but also the voltage distribution in the output transmission line region are strongly influenced by the driven sequence of the induction cells. The last three cavities were fired nearly $7 \mathrm{~ns}$ later than the ideal values. Thus, they show a low voltage adding efficiency. Besides, the electromagnetic wave will undergo multiple reflections in the output transmission line that serves as an inductor. The peak voltage gradually decreases along the line because of the inductive drop. Since the impedances of the output transmission line rise step by step, the inductive voltage drops also increase at the second group of three induction cells.

The voltage and current downstream of the voltage adder system, and the load voltage, are exhibited in Fig. 13.

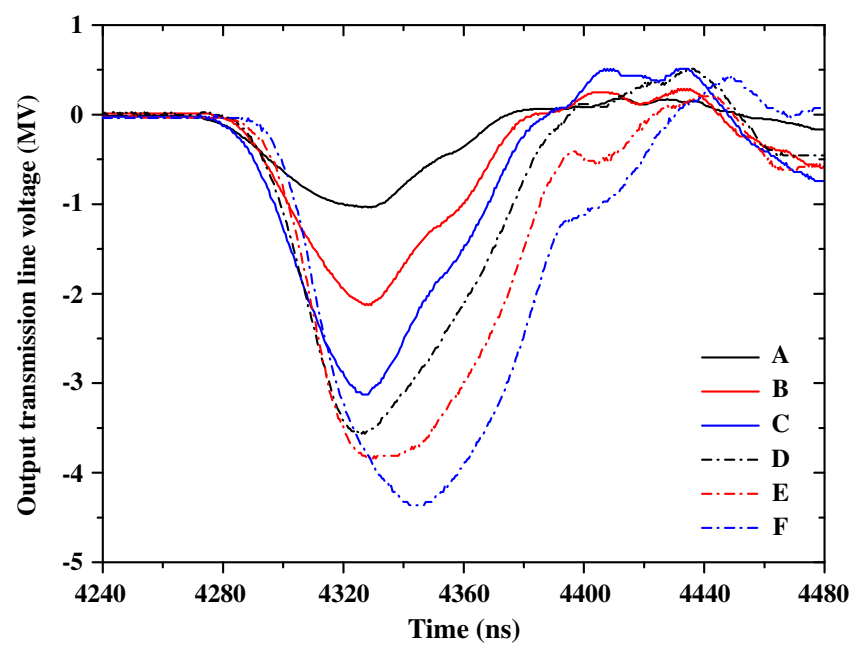

FIG. 12. Voltage waveforms acquired in the output transmission line region. The positions of the D-dot monitors are depicted in Fig. 8. 


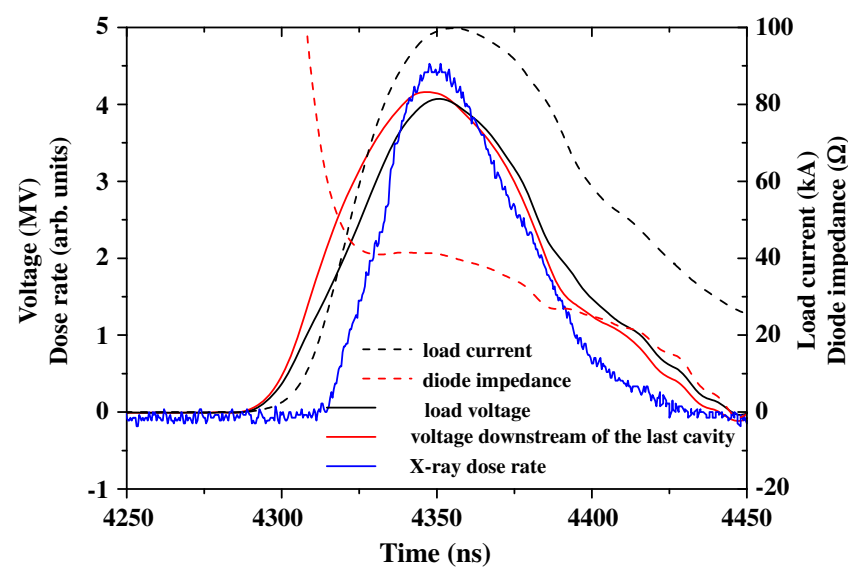

FIG. 13. The load current (black dashed line), RPD impedance (red dashed line), load voltage (black solid line), voltage downstream of the last cavity (red solid line), and X-ray dose rate (blue solid line). The load voltage is determined by induction correction from the voltage pulse obtained downstream of the last cavity. Since the RPD is close to the position of the B-dot sensor depicted in Fig. 8, the current waveform given by this monitor is regarded as the load current. Its amplitude was $100 \mathrm{kA}$.

The amplitudes of the voltage at the end of the last induction cavity and the load voltage were 4.2 and 4.1 MV, respectively. The $10-90 \%$ rise time of the load voltage pulse was about $38 \mathrm{~ns}$ and longer than those of voltage pulses feeding the induction cells. The increase of the rise time results from the equivalent inductance produced by the induction cavities and the output transmission line. Besides, previous investigations suggest that the unavoidable nonideal driven orders of the cells will also prolong the rise time [35]. The time history of the RPD impedance and the X-ray source dose rate are shown in Fig. 13 too. The diode impedance was roughly stable and equal to $40 \Omega$ during the period when the voltage pulse approached its maximum value. This impedance almost matches the summed impedance of the six WTLs. The full width at half maximum of the X-ray pulse was about $45 \mathrm{~ns}$ which was detected by silicon photodiodes locating $5 \mathrm{~m}$ from the source and on the forward radiographic axis.

For this shot, the X-ray dose at $1 \mathrm{~m}$ from the diode was $17.9 \mathrm{rad}$. It is determined by averaging the results from four $\mathrm{LiF}$ thermoluminescent dosimeters (TLDs). The locations of the TLDs are shown in Fig. 14. They are about $25 \mathrm{~cm}$ from the RPD anode rod tip and at $8^{\circ}$ off the radiographic axis. Figure 14 also displays the layout of the setup for spot size measurement. A tungsten rolled edge is utilized to obtain the edge spread function of the X-ray source. The source is collimated by a stainless steel collimator before it transmits through the rolled edge and is recorded by the image plate. The size of the collimator is approximately $1 \mathrm{~m}$ in diameter, $30 \mathrm{~cm}$ in length, with a hole of $8 \mathrm{~cm}$ in diameter at the central region. The distance between the $\mathrm{X}$-ray source and rolled edge is $1.995 \mathrm{~m}$ while the image plate is about $2.131 \mathrm{~m}$ from the rolled edge. Figure 15

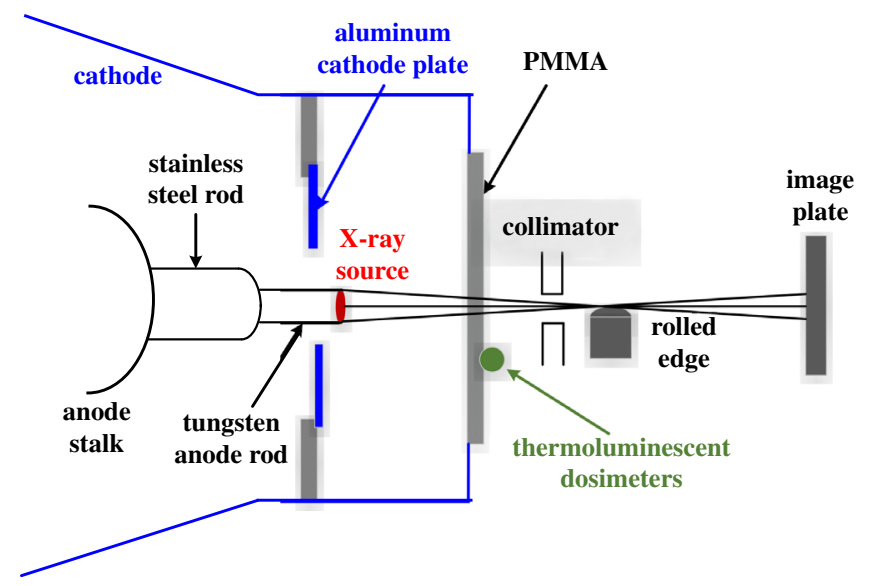

FIG. 14. Schematic of the X-ray dose and spot size measurements. There are four LiF TLDs utilized to monitor the X-ray dose for each shot. Those TLDs are sited into a small pillbox and attached to the PMMA endplate.

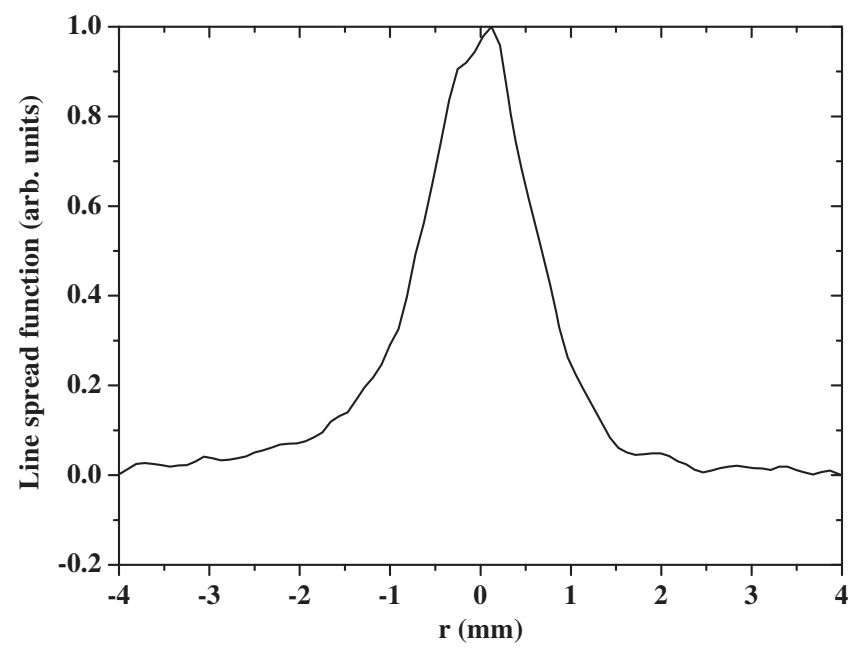

FIG. 15. Normalized LSF of the X-ray source. The spot size is defined as the FWHM of the LSF [41].

shows the derivative of the edge spread function which is also called the line spread function. The spot size was decided by the LSF and about $1.4 \mathrm{~mm}$.

\section{B. Operating reliability and synchronization ability}

We have executed 50 consecutive shots to evaluate the reliability of the Hawkeye-I. The capacitors charging voltages on those shots were $\pm 55 \mathrm{kV}$. The amplitudes of the voltage pulses downstream of the last induction cavity are presented in Fig. 16. The average value was 3.77 MV and the variations were within $0.15 \mathrm{MV}$. The X-ray doses at $1 \mathrm{~m}$ from the RPD of the 50 shots are also displayed in Fig. 16. The mean value was $15.4 \mathrm{rad}$ and the standard deviation [42] was $0.57 \mathrm{rad}$.

Shot 234 was a failure because of the prefire of one three-electrode gas switch. Since this shot, the air pressures 


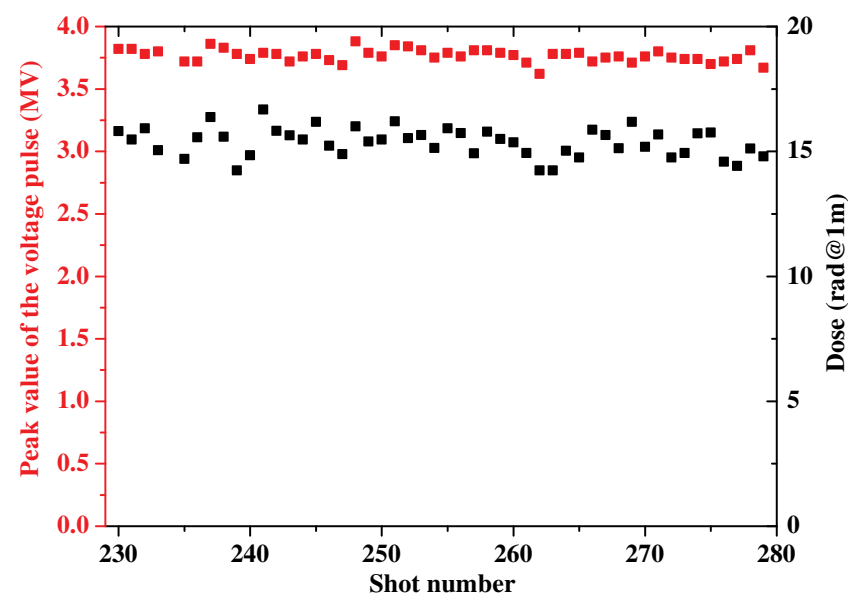

FIG. 16. Peak values of the voltage pulses downstream of the last cavity and the X-ray doses for 50 consecutive shots.

in the six three-electrode gas switches have been increased from 0.25 MPa to 0.4 MPa. These switches have worked successfully over 300 shots and the prefire has never occurred again. The working ratios of the gas switches are only $30 \%$ which are well less than those of switches employed in the linear transformer driver and Marx generator. Sufficiently degrading the work ratio is owing to the following reasons. The prefire of the three-electrode gas switch will result in charging the ISC unexpectedly which might induce the conduction of the LTGS and destroy the RPD. It leads to the failure of the hydrodynamic experiment thoroughly. Moreover, the unanticipated charging ISC may damage the tesla transformer and ISC in the event that the conduction of the LTGS does not take place. This is due to that the energy released from the capacitors propagates back and forth between the tesla transformer and ISC. It is very harmful to the plastic diaphragms of the two components. Although a sufficiently low working ratio

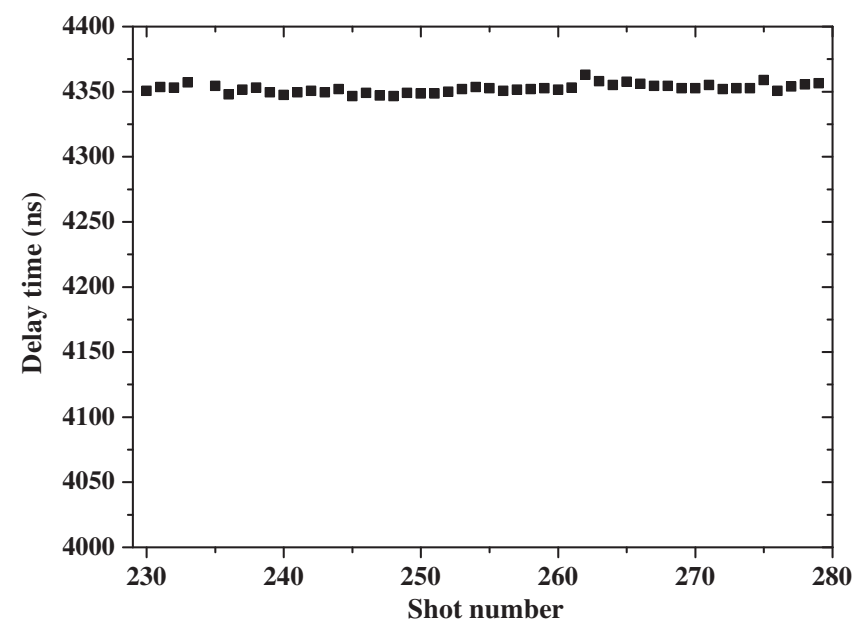

FIG. 17. Statistical delay times of Hawkeye-I for the 50 consecutive shots except for the failure one. might result in no-fire of the gas switch, the load voltage of about 2.8 MV could be obtained in case five stages of Hawkeye-I operate normally. On this condition, the image of the explosive event could still be acquired in despite of slightly inferior quality.

The delay time of the Hawkeye-I defining as the interval between the arrival of the laser beam for controlling the gas switch in the trigger unit and the onset of the X-ray pulse is also calculated. Since the zero start point of the dose rate waveform is unable to be acquired exactly, the time corresponding to $10 \%$ of the $\mathrm{X}$-ray dose maximum value is considered as the beginning of the X-ray pulse. The statistical delay times of the 50 shots are shown in Fig. 17. The mean value was $4352 \mathrm{~ns}$, and the standard deviation was $3.4 \mathrm{~ns}$ which was well less than the random uncertainty of the actions of explosion.

\section{CONCLUSIONS}

A pulsed power-driven X-ray source which is called Hawkeye-I, has been developed. It is able to produce a maximum output voltage of about $4.2 \mathrm{MV}$ at $\pm 60 \mathrm{kV}$ charging voltages. In this case, the radiation dose and spot size of the X-ray source are $17.9 \mathrm{rad}$ and $1.4 \mathrm{~mm}$, respectively. The operating reliability and synchronization ability of the facility are evaluated by executing 50 consecutive shots at $\pm 55 \mathrm{kV}$ charging voltages. The Hawkeye-I is capable of performing 98\% success shot. The standard deviation of the delay times defining as the interval between the arrival of the laser beam for controlling the trigger unit and the onset of the X-ray pulse signal is only $3.4 \mathrm{~ns}$.

The whole energy storage sections of Hawkeye-I include twelve capacitors and six gas switches, which are significantly fewer than other pulsed power facilities driven by Marx generators. Since the failures of gas switches and capacitors, such as prefire and no-fire of gas switches and short circuit of capacitors, are inherently inevitable, the fewer number of components utilized is helpful to enhance the reliability of the machine. Although the six threeelectrode gas switches work at very low working ratios and their jitters are about $10 \mathrm{~ns}$, the timing of the $\mathrm{X}$-ray pulse is not strongly related to the actions of those switches. The beginning of the X-ray source is mainly determined by the behaviors of the six LTGSs and the switch in the trigger unit. Since not only the switches triggered by laser beams could act accurately, the laser facilities and the three DDGs controlling the Nd:YAG lasers also work quite precisely, the Hawkeye-I is able to control the timing of the X-ray pulse accurately.

\section{ACKNOWLEDGMENTS}

This work was partially supported by the National Natural Science Foundation of China (Grants No. 51907181 and No. 51977201) and the Innovation Foundation of China Academy of Engineering Physics (Grant No. CX2019005). 
[1] J. Maenchen, G. Cooperstein, J. O'Malley, and I. Smith, Advances in pulsed power-driven radiography systems, Proc. IEEE 92, 1021 (2004).

[2] G.S. Cunningham and C. Morris, The development of flash radiography, Los Alamos Sci. 28, 76 (2003).

[3] C. Ekdahl, Modern electron accelerators for radiography, IEEE Trans. Plasma Sci. 30, 254 (2002).

[4] I. D. Smith, V. L. Bailey, J. J. Fockler, J. S. Gustwiller, D. L. Johnson, J. E. Maenchen, and D. W. Droemer, Design of a radiographic integrated test stand (RITS) based on a voltage adder, to drive a diode immersed in a high magnetic field, IEEE Trans. Plasma Sci. 28, 1653 (2000).

[5] D. Weidenheimer, P. Corcoran, R. Altes, J. Douglas, H. Nishimoto, I. Smith, R. Stevens, D. L. Johnson, R. White, J. Gustwiller, J. E. Maenchen, P. Menge, R. Carlson, R. D. Fulton, G. Cooperstein, D. Droemer, and E. Hunt, Design of a driver for the Cygnus X-ray source, in Pulsed Power Conference (IEEE, Las Vegas, 2001).

[6] J. Smith, R. Carlson, R. Fulton, J. Chavez, P. Ortega, R. O'Rear, R. Quicksilver, B. Anderson, D. Henderson, C. Mitton, R. Owens, S. Cordova, J. Maenchen, I. Molina, D. Nelson, and E. Ormond, Cygnus dual beam radiography source, in Pulsed Power Conference (IEEE, Monterey, 2005).

[7] E. C. Ormond, S. R. Cordova, I. Molina, D. S. Nelson, J. R. Smith, G. D. Corrow, M. D. Hansen, D. J. Henderson, and C. V. Mitton, Cygnus diverter switch analysis, IEEE Trans. Plasma Sci. 36, 2554 (2008).

[8] T. J. Goldsack, T. F. Bryant, P. F. Beech, S. G. Clough, G. M. Cooper, R. Davitt, R. D. Edwards, N. Kenna, J. McLean, A. G. Pearce, M. J. Phillips, K. P. Pullinger, D. J. Short, M. A. Sinclair, K. J. Thomas, J. R. Threadgold, M. C. Williamson, and K. Krushelnick, Multimegavolt multiaxis high-resolution flash $\mathrm{x}$-ray source development for a new hydrodynamics research facility at AWE Aldermaston, IEEE Trans. Plasma Sci. 30, 239 (2002).

[9] K. Thomas et al., The MERLIN induction voltage adder radiographic accelerator, in Pulsed Power Conference (IEEE, Brighton, 2017).

[10] G. Raboisson, P. Eyl, M. Roche, C. Malaval, and A. Johan, Asterix, a high intensity x-ray generator, in Pulsed Power Conference (IEEE, Monterey, 1989).

[11] H. Wei, J. H. Yin, P. F. Zhang, F. J. Sun, A. C. Qiu, T. X. Liang, J. T. Zeng, X. F. Jiang, Z. G. Wang, J. Sun, W. Y. Liu, Q. F. Luo, W. D. Ding, and Y. X. Hu, Development of a 4-MV, 80-kA-induction voltage adder for flash X-ray radiography, IEEE Trans. Plasma Sci. 47, 5030 (2019).

[12] H. Wei, J. H. Yin, P. F. Zhang, F. J. Sun, A. C. Qiu, T. X. Liang, X. F. Jiang, Z. G. Wang, J. Sun, Q. F. Luo, H. L. Yang, W. B. Yao, H. Y. Jiang, and H. Y. Wu, Simulation, experiment, and performance of a $4 \mathrm{MV}$ induction voltage adder machine for flash x-ray radiography, Phys. Rev. Accel. Beams 24, 020402 (2021).

[13] J. Smith, D. Nelson, E. Ormond, S. Cordova, I. Molina, G. Corrow, M. Hansen, D. Henderson, S. Lutz, and C. Mitton, Cygnus performance in subcritical experiments, in Pulsed Power Conference (IEEE, Albuquerque, 2007).

[14] J. Smith, M. Garcia, E. Ormond, M. Parrales, P. Flores, K. Hogge, S. Huber, M. Misch, J. Perez, T. Romero, and H. Truong, Cygnus performance on seven subcritical experiments, in Pulsed Power Conference \& Plasma Science (IEEE, Orlando, 2019).

[15] J. R. Smith, M. R. Garcia, E. C. Ormond, M. F. Parrales, P. A. Flores, K. W. Hogge, S. R. Huber, S. E. Mitchell, J. R. Perez, T. A. Romero, and H. V. Truong, Cygnus performance on Vega, Los Alamos National Laboratory Report, Technical Report No. LA-UR-18-24549, 2018.

[16] D. S. Nelson, E. C. Ormond, I. Molina, S. R. Cordova, J. R. Smith, G. D. Corrow, M. D. Hansen, D. J. Henderson, and C. V. Mitton, Cygnus trigger system, IEEE Trans. Plasma Sci. 36, 2730 (2008).

[17] E. C. Ormond, M. F. Parrales, M. R. Garcia, J. R. Smith, P. H. Amos, K. W. Hogge, M. K. Misch, M. Mohammed, and H. V. Truong, Cygnus system timing, in Pulsed Power Conference \& Plasma Science (IEEE, Orlando, 2019).

[18] G. Cooperstein, J. R. Boller, R. J. Commisso, D. D. Hinshelwood, D. Mosher, P. F. Ottinger, J. W. Schumer, S. J. Stephanakis, S. B. Swanekamp, B. V. Weber, and F.C. Young, Theoretical modeling and experimental characterization of a rod-pinch diode, Phys. Plasmas 8, 4618 (2001)

[19] F. C. Young, R. J. Commisso, R. J. Allen, D. Mosher, S. B. Swanekamp, and G. Cooperstein, F. Bayol, P. Charre, A. Garrigues, C. Gonzales, F. Pompier, and R. Vezinet, Rodpinch diode operation at 2 to $4 \mathrm{MV}$ for high resolution pulsed radiography, Phys. Plasmas 9, 4815 (2002).

[20] B. Etchessahar et al., Study and optimization of negative polarity rod pinch diode as flash radiography source at 4.5 MV, Phys. Plasmas 19, 093104 (2012).

[21] P. R. Menge, D. L. Johnson, J. E. Maenchen, D. C. Rovang, B. V. Oliver, D. V. Rose, and D. R. Welch, Optimization of a rod pinch diode radiography source at $2.3 \mathrm{MV}$, Rev. Sci. Instrum. 74, 3628 (2003).

[22] D. Nelson, M. Burke, J. Chael, E. Ormond, S. Cordova, and I. Molina, Cygnus source emission, in Pulsed Power Conference (IEEE, Washington, 2009).

[23] K. D. Hahn, N. Bruner, M. D. Johnston, B. V. Oliver, T. J. Webb, D. R. Welch, S. R. Cordova, I. Crotch, R. E. Gignac, J. J. Leckbee, I. Molina, S. Portillo, J. R. Threadgold, and D. Ziska, Overview of self-magnetically pincheddiode investigations on RITS-6, IEEE Trans. Plasma Sci. 38, 2652 (2010).

[24] J. D. Douglass et al., $100 \mathrm{GW}$ linear transformer driver cavity: Design, simulations, and performance, Phys. Rev. Accel. Beams 21, 120401 (2018).

[25] Y. Zhao, W. P. Xie, J. H. Jiang, L. Chen, F. P. Feng, M. Wang, and Z. Wang, Replacement of Marx generator by tesla transformer for pulsed power system reliability improvement, IEEE Trans. Plasma Sci. 47, 574 (2019).

[26] H. Bluhm, Pulsed Power Systems: Principles and Applications (Springer, Berlin, 2006), ISBN -103-540-26137-0.

[27] V. Carboni, P. Corcoran, J. Douglas, I. Smith, D. Johnson, R. White, B. Altes, R. Stevens, H. Nishimoto, R. Carlson, J. Smith, P. Ortega, J. Chavez, J. Maenchen, E. Ormond, D. Nelson, D. Henderson, T. Helvin, V. Mitton, and B. Anderson, Pulse power performance of the Cygnus 1 and 2 radiography source, in Pulsed Power Conference (IEEE, Dallas, 2003).

[28] G. A. Mesyats, Pulsed power, (Springer, New York, 2005), ISBN: 0-306-48653-9. 
[29] J. J. Deng et al., From concept to reality-A review to the primary test stand and its preliminary application in high energy density physics, Matter Radiat. Extremes 1, 48 (2016).

[30] L. Chen et al., Development of a fusion-oriented pulsed power module, Phys. Rev. Accel. Beams 22, 030401 (2019).

[31] P. Flores, D. J. Henderson, D. E. Good, K. Hogge, C. V. Mitton, I. Molina, C. Naffziger, S. R. Codova, and E. C. Ormond, Anodizing of high electrically stressed components, in Pulsed Power Conference (IEEE, San Francisco, 2013).

[32] F. Guo, W. P. Xie, Z. Wang, J. H. Jiang, M. H. Xia, B. Wei, S. P. Feng, Y. Zhao, J. J. Kang, M. Wang, W. K. Zou, and L. Chen, Design of a 1-MV induction cavity and validation of the two-dimensional circuit model, Phys. Rev. Accel. Beams 22, 020401 (2019).

[33] I. Smith, P. Corcoran, V. Carboni, V. Bailey, H. Kishi, D. L. Johnson, J. Maenchen, I. Molina, R. Carlson, D. Fulton, K. Hahn, J. Smith, D. Droemer, K. Thomas, M. Phillips, S. Croxon, R. Forgan, and I. D. Smith, Induction voltage adder architectures and electrical characteristics, in Pulsed Power Conference (IEEE, Dallas, 2003).

[34] J. J. Ramirez, K. R. Prestwich, and I. D. Smith, Highpower, short-pulse generators based on induction voltage adders, Proc. IEEE 80, 946 (1992).

[35] I. D. Smith, Induction voltage adders and the induction accelerator family, Phys. Rev. ST Accel. Beams 7, 064801 (2004).
[36] T. A. Holt, R. J. Allen, R. C. Fisher, R. J. Commisso, and D. L. Johnson, Analysis of switch performance on the mercury pulsed-power generator, in Pulsed Power Conference (IEEE, Monterey, 2005).

[37] F. Guo, W. K. Zou, B. Y. Gong, J. H. Jiang, L. Chen, M. Wang, and W.P. Xie, Modeling power flow in the induction cavity with a two dimensional circuit simulation, Phys. Rev. Accel. Beams 20, 020401 (2017).

[38] R. A. Mahaffey, J. Golden, S. A. Goldstein, and G. Cooperstein, Intense electron-beam pinch formation and propagation in rod pinch diodes, Appl. Phys. Lett. 33, 795 (1978).

[39] R. J. Commisso, G. Cooperstein, D. D. Hinshelwood, D. Mosher, P. F. Ottinger, S. J. Stephanakis, S. B. Swanekamp, B. V. Weber, and F. C. Young, Experimental evaluation of a megavolt rod-pinch diode as a radiography source, IEEE Trans. Plasma Sci. 30, 338 (2002).

[40] L. D. Geng, Y. He, J. Q. Yuan, M. H. Wang, L. B. Cao, S. P. Feng, and W. P. Xie, Study on eccentricity effects of the rod-pinch diode radiography source, Rev. Sci. Instrum. 90, 023304 (2019).

[41] B. V. Weber et al., Radiographic properties of plasma-filled rod-pinch diodes, IEEE Trans. Plasma Sci. 36, 443 (2008).

[42] A. S. Richardson, J. C. Zier, J. T. Engelbrecht, S. B. Swanekamp, J. W. Schumer, D. Mosher, P. F. Ottinger, D. L. Duke, T. J. Haines, M. P. McCumber, and A. Gehring, Simulations of the generation and transport of a $5 \mathrm{MV}$ end-point $\mathrm{x}$-ray beam on a pulsed power generator, Phys. Rev. Accel. Beams 22, 050401 (2019). 